\title{
Management of Hepatitis C Virus Infection - New Era Has Been Started
}

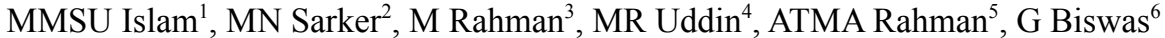

\begin{abstract}
:
Hepatitis $\mathrm{C}$ virus is a major cause of chronic hepatitis and is responsible for majority of liver transplantation worldwide. It has got no vaccine for prevention. Interferon containing regimen was the only management options for treatment of Hepatitis $\mathrm{C}$ virus till last year. Due to side effect profile and treatment cost this treatment options was not reachable for general population. Besides, response rate was not so satisfactory (Genotype $1,40-50 \%$, Genotype 2 \& $3,70-80 \%$ ). New oral antivirals were introduced in 2014 which has got little side effect profile as well as good treatment response rate (80-95\%). These new drugs with their different combination regimens along with indications, contraindications and treatment monitoring of Hepatitis $\mathrm{C}$ virus are discussed briefly in this review.
\end{abstract}

Key words: Hepatitis C Virus, Sustained Virologic Response, AntiVirals.

\section{Introduction:}

Hepatitis C Virus (HCV) infection is one of the main cause of chronic liver disease worldwide. It infects 170 million people worldwide and $1.6 \%$ of the population of the United States ${ }^{1}$. HCV prevalence in Europe is 1.03\%, Africa 5.3\%, Mediterranian region $4.6 \%$, Western pacific region $3.9 \%$ and South east Asia 2,13\% . In Bangladesh prevalence is $1 \%$ among health care workers ${ }^{3}$. About $55 \%$ to $85 \%$ of acutely infected persons ultimately lead to chronic infection ${ }^{4}$. Chronic HCV infection can lead to cirrhosis and hepatocellular carcinoma \& Chronic $\mathrm{HCV}$ is the only chronic viral infection that can be cured by antiviral therapy ${ }^{4}$. Till recently, interferon (INF) and Ribavirin (RBV) was the treatment options for $\mathrm{HCV}$ with a sustained virologic response (SVR) rate of $40 \%$ to $50 \%$ for genotype 1 and $70 \%$ to $80 \%$ for genotype 2 and 3 infection ${ }^{4}$. In 2011 , two protease inhibitor (direct acting antiviral) Telapravir and Bocepravir were licensed for Genotype 1 infection

1. Dr. M. M. Shahin-Ul-Islam, FCPS (Medicine), MD (Gastroenterology), Assistant Professor, Department of Gastroenterology, Faridpur Medical College, Faridpur.

2. Dr. Mst. Naznin Sarker, FCPS (Paediatrics), Junior Consultant, 250 bade General Hospital, Gopalgonj.

3. Dr. Mahabub Rahman, FCPS (Medicine), MD (Gastroenterology), Associate Professor (CC), Department of Gastroenterology, IBN Sina Medical College, Dhaka.

4. Dr. Md. Royes Uddin, FCPS (Medicine), MD (Gastroenterology), Assistant Professor, Department of Gastroenterology, Faridpur Medical College, Faridpur.

5. Dr. ATM Ataur Rahman, MD (Gastroenterology), Assistant Professor, Department of Gastroenterology, Faridpur Medical College, Faridpur.

6. Dr. Gonopati Biswas, DDS, Associate Prof, D Faridpur Medical College, Faridpur \& Superintendent, Faridpur Medical College, Hospital, Faridpur.

Address of correspondence :

Dr. M.M. Shahin-Ul-Islam, FCPS (Medicine), MD (Gastroenterology),

Assistant Professor, Department of Gastroenterology, Faridpur

Medical College, Faridpur. Mobile No: +8801711986290 ,

Email: shahin52dmc@gmail.com. in combination with INF \& RBV with a SVR rate 65$75 \%$ but due to side effect profile and cost they didn't became so popular ${ }^{5}$. In 2014, Sofosbuvir, Simepravir, Daclastavir was introduced with a SVR rate $80-95 \%$ with different combination \& genotype ${ }^{5}$. In addition, Ledipasvir, Paritaprevir, Ombitasvir, Ritonavir \& Dasabuvir has got excellent activity against HCV infection with a high SVR rate in various combinations. So, now HCV infection can be confidently treated with various INF free regimens also \& obviously new era has been started in the treatment of HCV infection. Intent of this review is to simplify various treatment modalities of HCV for easy understanding of our physician to deal with HCV infection.

\section{Management of $\mathrm{HCV}$ infection:}

\section{Goals and endpoints of $\mathrm{HCV}$ therapy:}

The goal of therapy is to cure HCV infection in order to prevent hepatic cirrhosis, decompensation of cirrhosis, HCC, severe extra-hepatic manifestations and death.

The endpoint of therapy is an SVR, defined by undetectable HCV RNA 12 weeks (SVR12) or 24 weeks (SVR24) after the end of therapy, as assessed by a sensitive molecular method with a lower limit of detection $\leq 15 \mathrm{IU} / \mathrm{ml}$.

Both SVR12 and SVR24 have been accepted as endpoints of therapy by regulators in the US and Europe. Long-term follow-up studies have shown that an SVR corresponds to a definitive cure of $\mathrm{HCV}$ infection in more than $99 \%$ of cases. In patients with advanced fibrosis and cirrhosis, HCV eradication 
reduces the rate of decompensation and will reduce, albeit not abolish the risk of HCC. In these patients surveillance for HCC should be continued. In patients with decompensated cirrhosis, $\mathrm{HCV}$ eradication reduces the need for liver transplantation. Whether HCV eradication impacts mid- to long-term survival in these patients is unknown.

\section{Pre-therapeutic assessment:}

The causal relationship between $\mathrm{HCV}$ infection and liver disease should be established, liver disease severity must be assessed, and baseline virological parameters should be determined. Search for other causes of chronic liver disease, or factors which are likely to affect the natural history or progression of liver disease, should be systematically investigated and all patients should be tested for other hepatotropic viruses, particularly hepatitis B virus (HBV), and for human immunodeficiency virus (HIV). Possible comorbidities, including alcoholism, autoimmunity, genetic or metabolic liver diseases (for instance genetic hemochromatosis, diabetes or obesity) and the possibility of drug-induced hepatotoxicity should be assessed.

\section{Assessment of liver disease severity}

Assessment of liver disease severity is recommended prior to therapy. Identifying patients with cirrhosis or advanced (bridging) fibrosis is of particular importance, as the post-treatment prognosis depends on the stage of fibrosis. Assessment of the stage of fibrosis is not required in patients with clinical evidence of cirrhosis. Patients with cirrhosis need surveillance for HCC. Since significant fibrosis may be present in patients with repeatedly normal ALT, evaluation of disease severity should be performed regardless of ALT levels. Fibrosis stage can be assessed by non-invasive methods initially, with liver biopsy reserved for cases where there is uncertainty or potential additional aetiologies.

\section{HCV RNA detection/quantification and genotype determination}

HCV RNA detection/quantification is indicated for the patient who may undergo antiviral treatment. HCV RNA quantification should be made by a reliable sensitive assay with a lower limit of detection of $\leq 15$ $\mathrm{IU} / \mathrm{ml}$, and HCV RNA levels should be expressed in $\mathrm{IU} / \mathrm{ml}$. The HCV genotype, including genotype 1 subtype, should alsobe assessed prior to treatment initiation. Genotyping/subtyping should be performed with an assay that accurately discriminates subtype la from 1b. HCV resistance testing should not be performed prior to therapy, because the SVR rates are very high both in patients without and with detectable amounts of resistance-associated variants.
Indications for treatment: Who should be treated and when?:

\section{Treatment is indicated}

* All treatment-naive and treatment-experienced patients with compensated and decompensated liver disease.

\section{Treatment should be prioritized}

* Patients with significant fibrosis (F3) or cirrhosis (F4), including decompensated cirrhosis.

* Patients with HIV co-infection.

* Patients with HBV co-infection.

* Patients with an indication for liver transplantation.

* Patients with HCV recurrence after liver transplantation.

* Patients with clinically significant extra-hepatic manifestations (Type 2 or 3 essential mixed cryoglobulinemia with endorgan manifestations like vasculitis, proteinuria, nephrotic syndrome, or membranoproliferative glomerulonephritis).

* Patient with Non Alcoholic Steatohepatitis (NASH).

* Patients with type 2 diabetes mellitus.

* Patients with debilitating fatigue.

* Individuals at risk of transmitting HCV (active injection drug users, men who have sex with men with high-risk sexual practices, women of childbearing age who wish to get pregnant, haemodialysis patients, incarcerated individuals).

\section{Treatment is justified}

* Patients with moderate fibrosis (F2)

\section{Treatment can be deferred}

* Patients with no or mild disease (F0-F1) and none of the above-mentioned extrahepatic manifestations.

\section{Treatment is not recommended}

* Patients with limited life expectancy due to nonliver related co-morbidities.

\section{Contra-indications to therapy:}

\section{IFN- $\alpha$ and Ribavirin}

This regimen is absolutely contra-indicated in the following patient groups: uncontrolled depression, psychosis or epilepsy; pregnant women or couples unwilling to comply with adequate contraception; severe concurrent medical diseases and co-morbidities including retinal disease, autoimmune thyroid disease; decompensated liver disease. The use of PegIFN- $\alpha$ is not recommended in patients with absolute neutrophil counts $<1500 / \mathrm{mm}^{3}$ and/or platelet counts $<90,000 / \mathrm{mm}^{3}$. Treatment of patients with advanced liver disease 
whose parameters fall outside of label recommendations may be feasible in experienced centres under careful monitoring and informed consent.

\section{Approved Direct-Acting Antivirals (DAAs)}

No absolute contra-indications to the DAAs exist till date. Caution is required with the use of Sofosbuvir in patients with severe renal impairment. The combination of Ritonavir-boosted Paritaprevir, Ombitasvir, and Dasabuvir is undergoing evaluation in patients with Child-Pugh B decompensated cirrhosis and is contra-indicated in patients with Child-Pugh C decompensated cirrhosis. Studies are on-going to assess the pharmacokinetics and safety of Simeprevir in decompensated cirrhosis.

\section{Treatment options:}

\section{Genotype 1:}

Any one of the following

1. Combination of weekly PegIFN- $\alpha$, daily weight based Ribavirin (1000 or $1200 \mathrm{mg}$ in patients $<75$ $\mathrm{kg}$ or $\geq 75 \mathrm{~kg}$, respectively), and daily Sofosbuvir (400 mg) 12 weeks.

2. Combination of weekly PegIFN- $\alpha$, daily weight based Ribavirin, and daily Simeprevir (150mg) for 12 weeks, PegIFN- $\alpha$ and Ribavirin for an additional 12 weeks (total treatment duration 24 weeks) in treatment-naive and prior relapser patients, including cirrhotic patients, and for an additional 36 weeks (total treatment duration 48 weeks) in prior partial and null responders, including cirrhotic patients.

3. Fixed-dose combination of Sofosbuvir (400mg) and Ledipasvir (90mg) in a single tablet administered once daily. Patients without cirrhosis (treatmentnaïve/treatment experienced) for 12 weeks. Patients with compensated cirrhosis (treatmentnaïve/treatment-experienced) with additional daily weight-based Ribavirin. Patients with compensated cirrhosis with contraindications to the use of Ribavirin or with poor tolerance to Ribavirin for 24 weeks without Ribavirin.

4. Combination of Ombitasvir (150mg), Paritaprevir (25mg), Ritonavir (100mg) and Dasabuvir (250 $\mathrm{mg}$ ). Patients with subtype $1 \mathrm{~b}$ without cirrhosis for 12 weeks, subtype $1 \mathrm{~b}$ with cirrhosis for 12 weeks with daily weight based Ribavirin, with subtype 1a without cirrhosis for 12 weeks with daily weightbased Ribavirin, with subtype 1a with cirrhosis for 24 weeks with daily weight based Ribavirin.

5. Combination of daily Sofosbuvir $(400 \mathrm{mg})$ and daily Simeprevir (150mg) for 12 weeks, adding daily weight-based Ribavirin in patients with cirrhosis. In patients with cirrhosis with contra-indications to Ribavirin, treatment for 24 weeks.

6. Combination of daily Sofosbuvir $(400 \mathrm{mg})$ and daily Daclastavir (60mg) for 12 weeks, adding daily weight-based Ribavirin in patients with cirrhosis. In patients with cirrhosis with contraindications to Ribavirin, treatment for 24 weeks.
Genotype 2:

Any one of the following

1. Must be treated with daily weight-based Ribavirin and daily Sofosbuvir (400mg) for 12 weeks without cirrhosis for 16 or 20 weeks with cirrhosis.

2. Cirrhotic and/or treatment-experienced patients can be treated with weekly PegIFN- $\alpha$, daily weightbased Ribavirin and daily Sofosbuvir (400mg) 12 weeks.

3. Cirrhotic and/or treatment-experienced patients can be treated with combination of daily Sofosbuvir (400mg) and daily Daclastavir (60mg) for 12 weeks.

Genotype 3:

Any one of the following

1. Combination of weekly PegIFN- $\alpha$, daily weight based Ribavirin and daily Sofosbuvir (400mg) 12 weeks.

2. Weight-based Ribavirin, and daily Sofosbuvir (400mg) for 24 weeks.

3. Without cirrhosis combination of daily Sofosbuvir (400mg) and daily Daclastavir (60mg) for 12 weeks, with cirrhosis this combination with weight-based Ribavirin for 24 weeks.

\section{Genotype 4:}

\section{Any one of the following}

1. Combination of weekly PegIFN- $\alpha$, daily weight based Ribavirin and daily Sofosbuvir (400mg) 12 Weeks.

2. Combination of weekly PegIFN- $\alpha$, daily weight based Ribavirin and daily Simeprevir (150mg) for 12 weeks then PegIFN- $\alpha$ and Ribavirin for an additional 12 weeks (total treatment duration 24 weeks) in treatment-naive and prior relapser patients, including cirrhotic patients, an additional 36 weeks (total treatment duration 48 weeks) in prior partial and null responders, including cirrhotic patients.

3. Fixed-dose combination of Sofosbuvir (400mg) and Ledipasvir (90mg) in a single tablet administered once daily. Patients without cirrhosis (treatmentnaïve/treatment-experienced patients) for 12 weeks. With compensated cirrhosis (treatmentnaïve/treatment-experienced patients with additional daily weight-based Ribavirin for 12 weeks. Patients with compensated cirrhosis with contraindications to the use of Ribavirin or with poor tolerance to Ribavirin for 24 weeks without Ribavirin.

4. Without cirrhosis fixed-dose combination of Ombitasvir (75mg), Paritaprevir (12.5mg) and Ritonavir (50mg) in one single tablet (two tablets once daily with food), for 12 weeks with daily weight-based Ribavirin. Patients with cirrhosis for 24 weeks. 
5. Combination of daily Sofosbuvir $(400 \mathrm{mg})$ and daily Simeprevir (150mg) 12 weeks, adding daily weight-based Ribavirin for patients with cirrhosis. In patients with cirrhosis with contra-indications to the use of Ribavirin, extending duration of treatment to 24 weeks must be considered.

6. Combination of daily Sofosbuvir (400mg) and daily Daclastavir (60mg) for 12 weeks, adding daily weight-based Ribavirin for patients with cirrhosis. In patients with cirrhosis with contra-indications to the use of Ribavirin, extending duration of treatment to 24 weeks must be considered.

7. Daily Sofosbuvir (400mg) and weight-based Ribavirin for 24 weeks

\section{Genotype 5/6:}

Any one of the following

1. Combination of weekly PegIFN- $\alpha$, daily weightbased Ribavirin and daily Sofosbuvir (400mg) 12 weeks.

2. Fixed-dose combination of Sofosbuvir $(400 \mathrm{mg})$ and Ledipasvir (90mg) in a single tablet administered once daily. Patients without cirrhosis (treatmentnaïve/treatment-experienced) for 12 weeks, with compensated cirrhosis (treatment-naïve/treatmentexperienced) for 12 weeks with daily weight-based Ribavirin. With compensated cirrhosis with contraindications to the use of Ribavirin or with poor tolerance to Ribavirin for 24 weeks without Ribavirin.

3. Combination of daily Sofosbuvir (400mg) and daily Daclastavir (60mg) for 12 weeks, adding daily weight-based Ribavirin for patients with cirrhosis. In patients with cirrhosis with contra-indications to the use of Ribavirin, extending duration of treatment to 24 weeks must be considered.

4. Weekly PEG-IFN plus weight-based Ribavirin for 48 weeks

\section{Decompensated Cirrhosis:}

1. Patients with decompensated cirrhosis (Child-Pugh $\mathrm{B}$ and Child-Pugh $\mathrm{C}$, up to 12 points) not on the waiting list for liver transplantation and without concomitant co-morbidities that could impact their survival can be treated with the combination of Sofosbuvir and Ribavirin for 16-20 weeks (genotype 2), the fixed-dose combination of Sofosbuvir and Ledipasvir (genotypes 1, 4, 5 and 6 ), or the combination of Sofosbuvir and Daclastavir (all genotypes), with weight-based Ribavirin for 12 weeks.

2. Patients with decompensated cirrhosis with contraindications to the use of Ribavirin or with poor tolerance to Ribavirin on treatment should receive the fixed-dose combination of Sofosbuvir and Ledipasvir (genotypes 1, 4, 5 or 6), or the combination of Sofosbuvir and Daclastavir (all genotypes) for 24 weeks without Ribavirin.

3. For genotype $1 \& 4$ daily fixed-dose combination Ledipasvir (90mg)/Sofosbuvir (400mg) and RBV (initial dose of $600 \mathrm{mg}$, increased as tolerated) for 12 weeks or for patients with anemia or RBV intolerance, daily fixed-dose combination Ledipasvir (90mg)/Sofosbuvir (400mg) for 24 weeks.

4. For genotype 2 or 3 daily Sofosbuvir (400mg) and weight-based Ribavirin (with consideration of the patient's creatinine clearance rate and hemoglobin level) for up to 48 weeks.

\section{Hepatocellular Carcinoma:}

Although the long-term benefit of antiviral therapy to reduce the risk of $\mathrm{HCC}$ in patients undergoing resection or ablation for $\mathrm{HCV}$-associated $\mathrm{HCC}$ is unknown, these patients frequently have advanced fibrosis and should receive appropriate antiviral therapy for their liver disease, following the guidelines above.

\section{HBV co-infection:}

1. Patients should be treated with the same regimens, following the same rules as HCV monoinfected patients.

2. If HBV replicates at significant levels before, during or after HCV clearance, concurrent HBV nucleoside/nucleotide analogue therapy is indicated.

\section{Management of Acute HCV Infection:}

Most patients with acute hepatitis $\mathrm{C}$ are asymptomatic, but a high rate of chronicity is expected without treatment (50-90\%). Symptomatic disease with jaundice, female gender and young age has been associated with spontaneous viral clearance, but none of these parameters accurately predicts spontaneous resolution at the individual level. Patients with acute hepatitis $\mathrm{C}$ should be considered for antiviral therapy in order to prevent progression to chronic hepatitis $\mathrm{C}$. The ideal time point for starting therapy has not been firmly established. Some investigators estimate that the onset of ALT elevation, with or without clinical symptoms, may be the ideal time point for treatment. It has also been suggested that patients should be followed with 4weekly HCV RNA quantification and that only those who remain HCV RNA positive at 12 weeks from onset should be treated. 


\section{Treatment options:}

1. PegIFN- $\alpha$ monotherapy (PegIFN- $\alpha 2 \mathrm{a}, 180 \mu \mathrm{g} /$ week or PegIFN- $\alpha 2 \mathrm{~b}, 1.5 \mu \mathrm{g} / \mathrm{kg} /$ week) for 12 weeks, it will achieve SVR in as many as $90 \%$ of cases.

2. PegIFN- $\alpha$ (PegIFN- $\alpha 2 \mathrm{a}, 180 \mu \mathrm{g} /$ week or PegIFN$\alpha 2 \mathrm{~b}, 1.5 \mu \mathrm{g} / \mathrm{kg} /$ week) should be combined with daily weight based Ribavirin for 24 weeks who are HIV-coinfected.

3. Although no data is available yet, IFN-free regimens can be used in these patients as they are expected to achieve high SVR rates. The same doses and durations as for patients with chronic hepatitis $\mathrm{C}$ can be used, without Ribavirin.

4. There is no indication for antiviral therapy as postexposure prophylaxis in the absence of documented HCV transmission.

\section{Pregnancy:}

Women of childbearing potential and/or their male partners must use an effective form of contraception during Ribavirin-containing treatment and for a period of 6 months after the treatment has concluded.

\section{Renal failure:}

1. Simeprevir, Daclastavir, Ritonavir,Paritaprevir, Ombitasvir and Dasabuvir are cleared by hepatic metabolism and can be used in patients with severe renal disease.

2. Sofosbuvir should not be administered to patients with an $\mathrm{eGFR}<30 \mathrm{ml} / \mathrm{min} / 1.73 \mathrm{~m}^{2}$ or with end-stage renal disease until more data is available

\section{Treatment Monitoring:}

Clinic visits or telephone contact are recommended as clinically indicated during treatment to ensure medication adherence and to monitor for adverse events and potential drug-drug interactions with newly prescribed medications.

1. Complete blood count (CBC), creatinine level, calculated glomerular filtration rate (GFR), and hepatic function panel are recommended after 4 weeks of treatment and as clinically indicated. Thyroid-stimulating hormone (TSH) is recommended every 12 weeks for patients receiving IFN. More frequent assessment for drug-related toxic effects (eg, $\mathrm{CBC}$ for patients receiving $\mathrm{RBV}$ ) is recommended as clinically indicated.

2. A real-time PCR-based assay with a lower limit of detection of $\leq 15 \mathrm{IU} / \mathrm{ml}$ should be used to monitor HCV RNA levels during and after therapy.

3. In patients treated with the triple combination of PegIFN- $\alpha$, Ribavirin and Sofosbuvir for 12 weeks, HCV RNA should be measured at baseline and at weeks 4, 12 (end of treatment), and 12 or 24 weeks after the end of therapy.
4. In patients treated with the triple combination of PegIFN- $\alpha$, Ribavirin and Simeprevir (12 weeks plus 12 or 36 weeks of PegIFN- $\alpha$ and Ribavirin alone), HCV RNA should be measured at baseline, week 4, week 12, week 24 (end of treatment in treatmentnaive patients and prior relapsers), week 48 (end of treatment in prior partial and null responders), and 12 or 24 weeks after the end of therapy.

5 . In patients treated with an IFN-free regimen, $\mathrm{HCV}$ RNA should be measured at baseline, week 2 (assessment of adherence), week 4, week 12 or 24 (end of treatment in patients treated 12 or 24 weeks, respectively), and 12 or 24 weeks after the end of therapy.

6. Prompt discontinuation of therapy is recommended for any

a. 10-fold increase in alanine aminotransferase (ALT) activity at week 4

b. Or, any increase in ALT of less than 10-fold at week 4 that is accompanied by any weakness, nausea, vomiting, or jaundice, or accompanied by increased bilirubin, alkaline phosphatase, or international normalized ratio. Asymptomatic increases in ALT of less than 10-fold elevated at week 4 should be closely monitored and repeated at week 6 and week 8 .

\section{Stopping rules:}

1. With the triple combination of PegIFN- $\alpha$, Ribavirin and Simeprevir, treatment should be stopped if HCV RNA level is $\geq 25 \mathrm{IU} / \mathrm{ml}$ at treatment week 4 , week 12 or week 24 and immediate switch to another IFN-containing,direct acting antiviral containing or to an IFN-free regimen without a protease inhibitor should be considered.

2. No futility rules have been defined for other treatment Regimens

\section{Post-treatment follow up after achieving SVR:}

1. Non-cirrhotic patients with SVR should be retested for ALT and HCV RNA at 48 weeks posttreatment, then discharged if ALT is normal and HCV RNA is negative.

2. Cirrhotic patients, and probably also patients with advanced fibrosis (F3), with SVR should undergo surveillance for HCC every 6 months by means of ultrasound.

3. Following SVR, monitoring for $\mathrm{HCV}$ reinfection through annual HCV RNA assessment should be undertaken in people who inject drugs or men who have sex with men with on-going risk behavior. In such cases, a quantitative HCV RNA assay rather than an anti-HCV serology test is recommended.

4. A baseline endoscopy is recommended to screen for varices if cirrhosis is present. Patients in whom varices are found should be treated and followed up as indicated. 
5. Assessment of other causes of liver disease is recommended for patients who develop persistently abnormal liver tests after achieving an SVR.

Follow up of untreated patient and of patient with treatment failure:

1. Should be regularly followed up.

2. Noninvasive methods of fibrosis staging (elastography) 1 to 2 yearly.

3. Surveillance for hepatocellular carcinoma with ultrasound testing every 6 months is recommended for patients with advanced fibrosis (ie, Metavir stage F3 or F4) or cirrhosis.

4. Endoscopic surveillance for esophageal varices is recommended if cirrhosis is present.

5. Evaluation for retreatment is recommended as effective alternative treatments become available.

\section{Conclusion:}

With invent of new oral direct acting antiviral drugs for the management of HCV infection we are hopeful that our resource limited population can be treated effectively. Though all oral drugs are not available in our country (Only Ribavirin \&Sofosbuvir are available), we can hope that in the near future more drug will be available to combat our $\mathrm{HCV}$ infected patient.

\section{References :}

1. Armstrong GL, Wasley A, Simrad EP, et al. The prevalence of hepatitis C virus infection in the united states, 1999 through 2002. Ann Intern Med 2006;144:705-14.

2. WHO global surveillance and control of Hepatitis C. Report of WHO consultation organized in collaboration with the viral hepatitis prevention board, Antwerp, Belgium. J Viral Hept. 1999;6:35-47.

3. Uddin MR, Islam MMSU, Hossain MZ, Sarker MN, SalimullahASM,Begum F, et el. Seroprevalence of Hepatitis C Virus among Health Care Workers. 2009. J Dhaka Med Coll; 18(1):70-74.

4. Jacqueline G, O'Leary, Gray LD. Hepatitis C. In: Feldman M, Friedman LS, Brandt LJ,Editors. Sleisenger and Fordtran's Gastrointestinal and Liver Disease Pathophysiology/Diagnosis/ Mangement. 9thedition.Philadelphia: Elsevier;2010.p.1313.

5. Clinical Practice Guidelines. EASL recommendations on Treatment of Hepatitis C 2015. www.easl.eu>HEPC-2015>Fullreport.

6. AASLD. Recommendations for Testing, Managing, and Treating Hepatitis C. http://www.hcvguidelines.org. 